\title{
Selection of covariates
}

Strong correlations between pairs of the abiotic variables $(|\rho|>=0.75)$ are highlighted in Table S1. The four Local Moran's I of bathymetry variables were highly correlated $(\rho=1.0)$; as were the four Local Moran's I of backscatter variables $(\rho>=0.97)$ and the four TPI variables $(\rho>=0.89)$. This indicates that over the extent of the study area scale was not a significant issue for these three measures. Strong correlations were also found amongst the morphological parameters (slope, topographic relief and surface area) $(\rho>=0.75)$, between sediment sorting and a seabed exposure variable $(|\rho|=0.82)$, and between Fe and water temperature $(|\rho|=$ 0.79). The correlations between bathymetry and two seabed exposure variables $(\rho=$ 0.79) were expected because bathymetry was an input to the SWAN model. The strong correlations between skewness and kurtosis (measures of asymmetry) $(|\rho|=$ 0.93) and between mud and sand $(|\rho|=1.0)$ were also expected because they are interrelated descriptors of the grain size distribution for each sample.

The abiotic variables with $\mathrm{R}^{2}$ values greater than 0.05 for the response variables are listed in Tables S2 to S12. There were always several abiotic variables that met this criterion for each response variable. For example, Species Richness was most strongly related to the proportion of mud and sand $\left(\mathrm{R}^{2}>0.22\right)$; while the Shannon Index correlated best with TSM $\left(\mathrm{R}^{2}>0.20\right)$ (Tables S3 and S4). Among the species data, the best relationships were observed between the polychaete Diopatra dentata and seabed exposure $\left(\mathrm{R}^{2}>0.55\right)$ (Dist_160; Table S5), the bivalve Venerupis galactites and sorting $\left(\mathrm{R}^{2}>0.52\right)$ (Table $\left.\mathrm{S} 11\right)$, and the polychaetes Owenia australis and Mesochaetopterus minutus and rugosity $\left(\mathrm{R}^{2}>0.38 ; \mathrm{R}^{2}>0.45\right)(\mathrm{TR}$ _7 Table S8 and Slope_3; Table S9). 


\section{Table S1: High correlations between the abiotic variables}

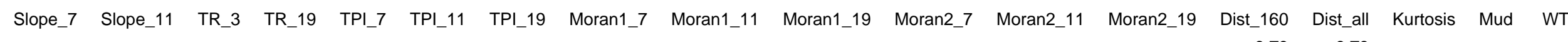

Bathymetry

Slope_3

Slope_7

Slope_19

TR 3

TR_11

SA 3

TPI_3

TPI_7

TPI_11

Moran1_3

Moran1_7

Moran1_11

Moran2_3

Moran2_7

Moran2_11

Dist_160

Dist_90

Dist_all

$\mathrm{Fe}$

Sorting

Skewness

Sand
0.75

0.85

$0.81 \quad 0.95$

0.80

$\begin{array}{lll}0.94 & 0.92 & 0.89 \\ & 0.99 & 0.96 \\ & & 0.98\end{array}$


Table S2: Results of the surrogacy analysis for the response variable of Total Abundance

\begin{tabular}{|c|c|c|c|c|c|}
\hline variable & $\mathrm{R}^{2(1)}$ & Selected $^{(2)}$ & $\mathrm{BDT}^{(3)}$ & $\mathrm{RFDT}^{(4)}$ & $\mathrm{DT}^{(5)}$ \\
\hline Mud & 0.2109 & yes & & 100 & \\
\hline Sand & 0.1985 & & & & \\
\hline Slope_3 & 0.175 & yes & & & 100 \\
\hline $\mathrm{K}_{\mathrm{d}}$ & 0.1364 & yes & 94 & 86 & \\
\hline Skewness & 0.1267 & yes & & & \\
\hline SA_7 & 0.1208 & yes & & & \\
\hline Chl-a & 0.1172 & yes & & & \\
\hline Gravel & 0.1054 & yes & & & \\
\hline TR_3 & 0.1025 & & & & \\
\hline Moran1_7 & 0.0964 & yes & & & \\
\hline Moran1_11 & 0.0961 & & & & \\
\hline Moran1_19 & 0.0945 & & & & \\
\hline Moran1_3 & 0.0942 & & & & \\
\hline Slope_7 & 0.0918 & yes & 64 & & \\
\hline Kurtosis & 0.0908 & & & & \\
\hline TS & 0.0891 & yes & 83 & 98 & \\
\hline DO & 0.0756 & yes & 72 & 87 & 27 \\
\hline MGS & 0.0663 & yes & & & \\
\hline Slope_11 & 0.0527 & & & & \\
\hline Aspect & & yes & & & \\
\hline Habitat & & yes & 100 & 96 & 42 \\
\hline
\end{tabular}

Note: (1) $\mathrm{R}^{2}$ values from the exploratory analysis; (2) whether the variable was selected after the selection of covariates process; (3) importance scores identified by the BDT; (4) importance scores identified by the RFDT; and (5) importance scores identified by the DT. 
Table S3: Results of the surrogacy analysis for the response variable of Species Richness

\begin{tabular}{|l|r|l|r|r|r|}
\hline variable & \multicolumn{1}{|l|}{$R^{2}$} & selected & BDT & RFDT & DT \\
\hline Mud & 0.2261 & yes & 98 & 99.5 & \\
\hline Sand & 0.2208 & & & & \\
\hline Chl-a & 0.1835 & yes & 95 & & \\
\hline TS & 0.1451 & yes & & 86 & 74 \\
\hline $\mathrm{K}_{\mathrm{d}}$ & 0.1437 & yes & & 65 & \\
\hline Backscatter & 0.1383 & yes & & & \\
\hline MGS & 0.1353 & yes & & & \\
\hline Fe & 0.1156 & yes & 87 & & \\
\hline CaCO3 & 0.1156 & yes & & & \\
\hline Kurtosis & 0.1029 & yes & & & \\
\hline Bathymetry & 0.0988 & yes & & 84 & 69 \\
\hline TSM & 0.0949 & yes & 83 & 68 & \\
\hline Moran2_7 & 0.0911 & yes & & & \\
\hline Skewness & 0.089 & & & & \\
\hline Moran2_3 & 0.0819 & & & & \\
\hline Sorting & 0.076 & yes & & & \\
\hline Moran1_3 & 0.063 & yes & & 70 & \\
\hline Moran1_11 & 0.063 & & & & \\
\hline Moran1_19 & 0.063 & & & & \\
\hline Moran1_7 & 0.0629 & & & & \\
\hline Dist_90 & 0.0578 & yes & & & \\
\hline PTCO 2 & 0.0563 & yes & & & \\
\hline SSA & 0.0562 & yes & & & \\
\hline TR_19 & 0.0543 & yes & & & \\
\hline SA_11 & 0.0529 & yes & 100 & 94 & \\
\hline Dist_160 & 0.0522 & & & & \\
\hline WT & 0.0501 & & & & \\
\hline Aspect & & yes & & & \\
\hline Habitat & & yes & 86 & 100 & 100 \\
\hline
\end{tabular}


Table S4: Results of the surrogacy analysis for the response variable of Shannon index

\begin{tabular}{|l|r|l|r|r|r|}
\hline variable & \multicolumn{1}{|l|}{$R^{2}$} & selected & BDT & RFDT & DT \\
\hline TSM & 0.2038 & yes & 76 & 58 & \\
\hline Gravel & 0.1653 & yes & 85 & & \\
\hline Slope_7 & 0.1457 & yes & & & \\
\hline Slope_3 & 0.1425 & yes & & 76 & \\
\hline SA_11 & 0.1421 & yes & & 59 & 81 \\
\hline Sorting & 0.1332 & yes & & & \\
\hline Moran1_11 & 0.116 & yes & 100 & 100 & \\
\hline Moran1_7 & 0.1155 & & & & \\
\hline Slope_11 & 0.1141 & & & & \\
\hline Moran1_19 & 0.1141 & & & & \\
\hline Moran1_3 & 0.1127 & & & & \\
\hline TR_19 & 0.1016 & yes & & & \\
\hline Slope_19 & 0.0915 & & & & \\
\hline CaCO 3 & 0.0814 & yes & & & \\
\hline Dist_90 & 0.0781 & yes & & & \\
\hline pTCO 2 & 0.0688 & yes & 28 & & \\
\hline Backscatter & 0.0668 & yes & 55 & 37 & \\
\hline MGS & 0.064 & yes & & & \\
\hline Bathymetry & 0.0629 & yes & & & \\
\hline DO & 0.0607 & yes & & & \\
\hline SA_7 & 0.0599 & yes & & & \\
\hline TPI_7 & 0.0592 & yes & & & \\
\hline TR_7 & 0.0554 & yes & & & \\
\hline TPI_3 & 0.054 & & & & \\
\hline SA_19 & 0.0539 & yes & & & \\
\hline TR_3M & 0.0529 & & & & \\
\hline Dist_all & 0.0521 & & & & \\
\hline TPI_11 & 0.0503 & & & & \\
\hline Aspect & & yes & & 66 & \\
\hline Habitat & & & 35 & \\
\hline
\end{tabular}


Table S5: Results of the surrogacy analysis for the response variable of Diopatra dentata

\begin{tabular}{|l|r|l|r|r|r|}
\hline variable & $\mathrm{R}^{2}$ & Selected & BDT & RFDT & DT \\
\hline Dist_160 & 0.5576 & yes & 100 & 100 & 100 \\
\hline Fe & 0.5311 & yes & 47 & 66 & \\
\hline Skewness & 0.4945 & yes & & 79 & \\
\hline Bathymetry & 0.4893 & & & & \\
\hline Dist_all & 0.4443 & & & & \\
\hline Mud & 0.3916 & yes & & & 11 \\
\hline Kurtosis & 0.3087 & & & & \\
\hline Kd & 0.2973 & yes & & & \\
\hline Sorting & 0.2957 & yes & & & \\
\hline Moran1_19M & 0.2844 & yes & & & \\
\hline Moran1_7M & 0.2843 & & & & \\
\hline Moran1_11M & 0.2843 & & & & \\
\hline Moran1_3M & 0.2841 & & & & \\
\hline MGS & 0.2343 & yes & & & \\
\hline Sand & 0.1784 & & & & \\
\hline Backscatter & 0.1654 & yes & & & \\
\hline CaCO ${ }_{3}$ & 0.1654 & yes & & & \\
\hline Dist_90 & 0.1383 & yes & & & \\
\hline TSM & 0.1324 & yes & & 42 & \\
\hline SSA & 0.1127 & yes & & & \\
\hline TR_3 & 0.083 & yes & 30 & & \\
\hline pTCO 2 & 0.0784 & yes & & & \\
\hline WT & 0.053 & & & & \\
\hline Aspect & & yes & & & \\
\hline Habitat & & yes & & & \\
\hline
\end{tabular}


Table S6: Results of the surrogacy analysis for the response variable of Rhinocetes robustus

\begin{tabular}{|c|c|c|c|c|c|}
\hline variable & $\mathrm{R}^{2}$ & selected & BDT & RFDT & DT \\
\hline $\mathrm{CaCO}_{3}$ & 0.2547 & yes & 100 & 100 & 100 \\
\hline $\mathrm{K}_{\mathrm{d}}$ & 0.202 & yes & & 53 & 34 \\
\hline Dist_160 & 0.1751 & yes & 81 & & \\
\hline Mud & 0.1375 & yes & & & \\
\hline Kurtosis & 0.1262 & yes & & & \\
\hline Sorting & 0.1101 & yes & & & \\
\hline Skewness & 0.1014 & & & & \\
\hline $\mathrm{pTCO}_{2}$ & 0.0921 & yes & & & \\
\hline TS & 0.0884 & yes & & & \\
\hline Dist_all & 0.0874 & & & & \\
\hline TSM & 0.0872 & yes & & & \\
\hline MGS & 0.0841 & yes & & 22 & 0 \\
\hline TPI_3 & 0.0766 & yes & & & \\
\hline Bathymetry & 0.0762 & & & & \\
\hline TR_19 & 0.0634 & yes & & & \\
\hline Sand & 0.0544 & & & & \\
\hline TPI_11 & 0.0534 & & & & \\
\hline TPI_7 & 0.0522 & & & & \\
\hline TPI_19 & 0.051 & & & & \\
\hline Aspect & & yes & & & \\
\hline Habitat & & yes & & & \\
\hline
\end{tabular}

Table S7: Results of the surrogacy analysis for the response variable of Nephtyus inornata

\begin{tabular}{|l|r|l|r|r|r|}
\hline variable & \multicolumn{1}{|l|}{$\mathrm{R}^{2}$} & selected & BDT & RFDT & DT \\
\hline $\mathrm{K}_{\mathrm{d}}$ & 0.1489 & yes & 64 & 64 & \\
\hline Gravel & 0.0961 & yes & 77 & & 71 \\
\hline Moran2_19 & 0.0926 & yes & & & \\
\hline Moran2_11 & 0.087 & & & & \\
\hline Moran2_7 & 0.0803 & & & & \\
\hline TS & 0.0795 & yes & & & \\
\hline Moran2_3 & 0.0772 & & & & \\
\hline Moran1_19 & 0.07 & yes & & & \\
\hline Moran1_11 & 0.0697 & & & & \\
\hline Moran1_3 & 0.069 & & & & \\
\hline Moran1_7 & 0.069 & & & & \\
\hline SSA & 0.06 & yes & & & \\
\hline MGS & 0.058 & yes & & 68 & \\
\hline Slope_19 & 0.0566 & yes & & & \\
\hline Backscatter & 0.053 & yes & & & \\
\hline Aspect & & yes & & & \\
\hline Habitat & & yes & 100 & 100 & 100 \\
\hline
\end{tabular}


Table S8: Results of the surrogacy analysis for the response variable of Owenia australis

\begin{tabular}{|l|r|l|r|r|r|}
\hline variable & \multicolumn{1}{|l|}{$\mathrm{R}^{2}$} & selected & BDT & \multicolumn{1}{l|}{ RFDT } & DT \\
\hline TR_7 & 0.3838 & yes & & 23 & 18 \\
\hline Dist_90 & 0.382 & yes & & 38 & 21 \\
\hline Dist_all & 0.359 & & & & \\
\hline Dist_160 & 0.2303 & yes & 69 & & \\
\hline SA_7 & 0.2138 & yes & & & \\
\hline TR_19 & 0.1817 & yes & & & \\
\hline Mud & 0.1516 & yes & & & \\
\hline Sand & 0.1436 & & & & \\
\hline SA_11 & 0.136 & yes & 100 & 100 & 100 \\
\hline Bathymetry & 0.1249 & & & & \\
\hline Sorting & 0.0884 & yes & 19 & & \\
\hline WT & 0.0735 & yes & 20 & & \\
\hline Slope_11 & 0.0688 & yes & & 18 & \\
\hline Skewness & 0.0602 & yes & & & \\
\hline SA_19 & 0.0561 & yes & & & \\
\hline TR_3 & 0.0546 & yes & & & \\
\hline SA_3 & 0.0531 & & & & \\
\hline Aspect & & yes & & & \\
\hline Habitat & & yes & & & \\
\hline
\end{tabular}


Table S9: Results of the surrogacy analysis for the response variable of Mesochaetopterus minutes

\begin{tabular}{|c|c|c|c|c|c|}
\hline variable & $\mathrm{R}^{2}$ & Selected & BDT & RFDT & DT \\
\hline Slope 3 & 0.4564 & yes & & 100 & \\
\hline TR 3 & 0.307 & & & & \\
\hline Gravel & 0.2181 & yes & 94 & & \\
\hline Moran1_7 & 0.2117 & yes & 100 & 55 & \\
\hline Moran1_11 & 0.2115 & & & & \\
\hline Moran1_19 & 0.208 & & & & \\
\hline Moran1_3 & 0.2069 & & & & \\
\hline TSM & 0.1675 & yes & 59 & 43 & \\
\hline Slope_7 & 0.1053 & yes & & & \\
\hline Skewness & 0.1022 & yes & & 26 & \\
\hline SA_7 & 0.0792 & yes & & & \\
\hline TR_19 & 0.075 & yes & & & \\
\hline $\mathrm{pTCO}_{2}$ & 0.0744 & yes & & 26 & \\
\hline Slope_11 & 0.0703 & & & & \\
\hline SA_11 & 0.0698 & yes & & & \\
\hline DO & 0.0698 & yes & & & \\
\hline Kurtosis & 0.0633 & & & & \\
\hline TPI_7 & 0.0597 & yes & & & \\
\hline Sorting & 0.0529 & yes & & & \\
\hline Aspect & & yes & & 29 & 50 \\
\hline Habitat & & yes & & 16 & 100 \\
\hline
\end{tabular}

Table S10: Results of the surrogacy analysis for the response variable of Echinocardium cordatum

\begin{tabular}{|l|r|l|r|r|r|}
\hline variable & \multicolumn{1}{|l|}{$\mathrm{R}^{2}$} & selected & BDT & RFDT & DT \\
\hline TS & 0.2156 & yes & & & \\
\hline Sorting & 0.168 & yes & 100 & 100 & 100 \\
\hline pTCO $_{2}$ & 0.1363 & yes & & & 94 \\
\hline Backscatter & 0.1039 & yes & & 40 & \\
\hline Mud & 0.0953 & yes & & & \\
\hline Sand & 0.0851 & & & & \\
\hline TR_3 & 0.0606 & yes & & & \\
\hline DO & 0.0504 & yes & & & \\
\hline Aspect & & yes & & 48 & \\
\hline Habitat & & yes & 16 & & \\
\hline
\end{tabular}


Table S11: Results of the surrogacy analysis for the response variable of Venerupis galactites

\begin{tabular}{|l|r|l|l|l|l|}
\hline variable & \multicolumn{1}{|l|}{$\mathrm{R}^{2}$} & selected & BDT & RFDT & DT \\
\hline Sorting & 0.5244 & yes & & & 100 \\
\hline Moran2_3 & 0.2301 & yes & 100 & 100 & \\
\hline Dist_90 & 0.168 & yes & & & \\
\hline Moran2_7 & 0.1346 & & & & \\
\hline Dist_all & 0.1317 & & & & \\
\hline Moran2_11 & 0.1136 & & & & \\
\hline Moran2_19 & 0.1081 & & & & \\
\hline Dist_160 & 0.077 & yes & & & \\
\hline Aspect & & yes & & & \\
\hline Habitat & & yes & 84 & 40 & \\
\hline
\end{tabular}

Table S12: Unciolidae_sp1 (mean: 6.39; STD: 11.23)

\begin{tabular}{|c|c|c|c|c|c|}
\hline variable & $\mathrm{R}^{2}$ & selected & BDT & RFDT & DT \\
\hline $\mathrm{K}_{\mathrm{d}}$ & 0.2481 & yes & & & 100 \\
\hline $\mathrm{CaCO}_{3}$ & 0.2369 & yes & 100 & & \\
\hline Skewness & 0.203 & yes & 46 & 83 & \\
\hline Mud & 0.1787 & yes & & & \\
\hline Dist_160 & 0.1762 & yes & 25 & & \\
\hline $\mathrm{Fe}$ & 0.1706 & yes & 20 & & \\
\hline Sorting & 0.1642 & yes & & 64 & 47 \\
\hline Kurtosis & 0.159 & & & & \\
\hline MGS & 0.1409 & yes & & & \\
\hline $\mathrm{pTCO}_{2}$ & 0.1224 & yes & 39 & & \\
\hline Backscatter & 0.1032 & yes & 21 & & \\
\hline Dist_all & 0.1021 & & & & \\
\hline Sand & 0.0911 & & & & \\
\hline Moran1_3 & 0.086 & yes & 50 & & \\
\hline Moran1_19 & 0.086 & & & & \\
\hline Moran1_7 & 0.0859 & & & & \\
\hline Moran1_11 & 0.0859 & & & & \\
\hline Bathymetry & 0.0854 & & & & \\
\hline SSA & 0.0809 & yes & 23 & 100 & \\
\hline TR_19 & 0.0725 & yes & & 25 & 12 \\
\hline TSM & 0.0666 & yes & 34 & 30 & \\
\hline Aspect & & yes & 58 & & \\
\hline Habitat & & yes & & & \\
\hline
\end{tabular}



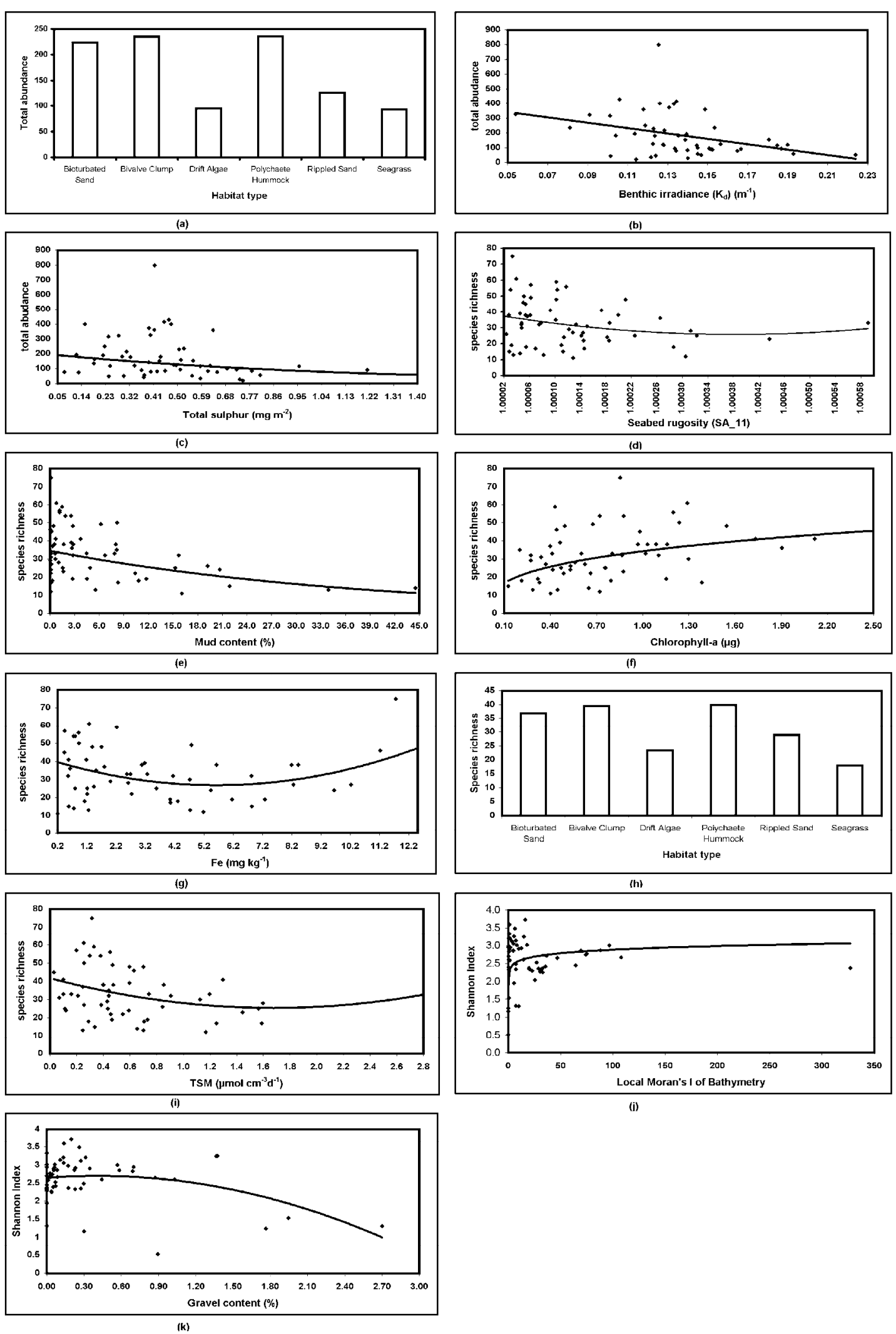

Figure S1: Sample values fitted with curves (see section 3.3.1) for biodiversity indices; (a): habitat type and mean total abundance; (b): $\mathrm{K}_{\mathrm{d}}$ and total abundance; (c): TS and total abundance; (d): SA_11 and species richness; (e): \%Mud and species richness; (f): Cholorphyll a and species richness; (g): $\mathrm{Fe}$ and species richness; (h): habitat type and species richness; (i): TSM and species richness; (j): local Morans I of bathymetry and Shannon index; (k): \%Gravel and Shannon index. 


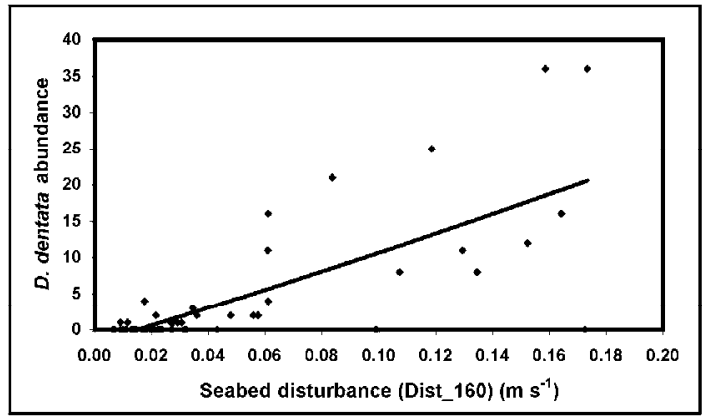

(a)

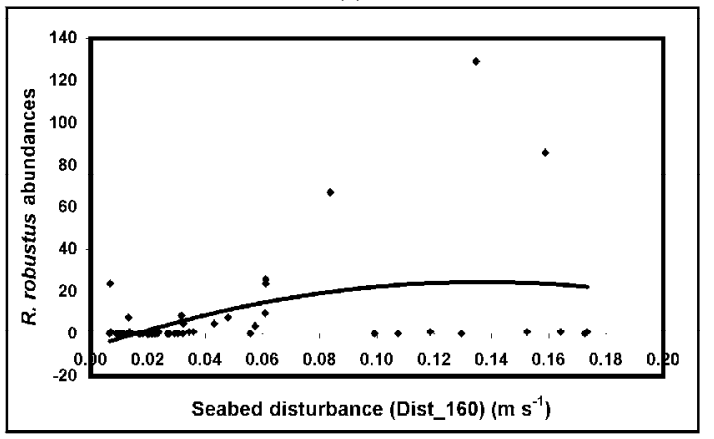

(c)

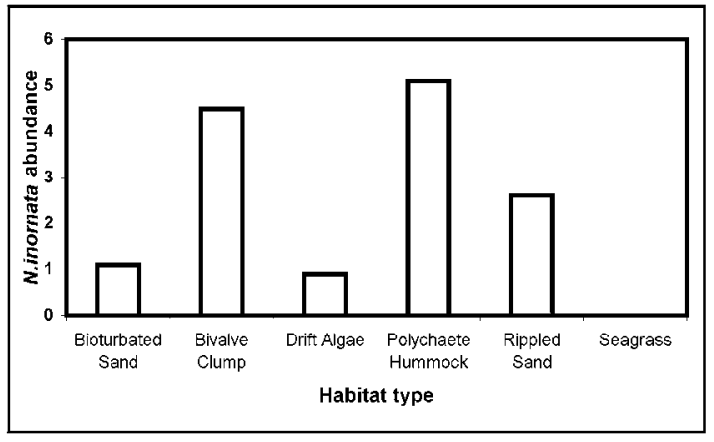

(e)

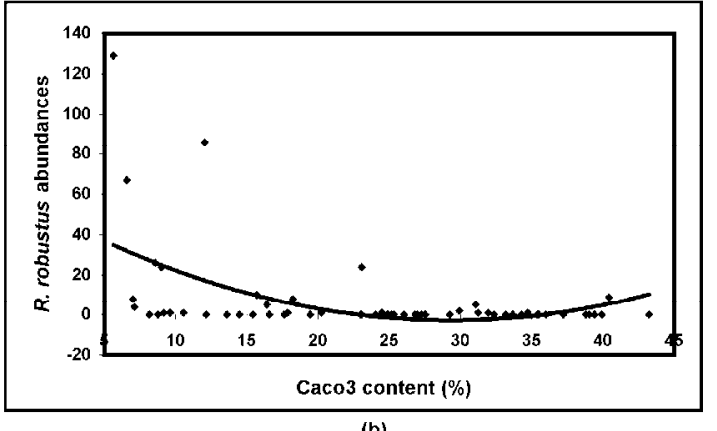

(b)

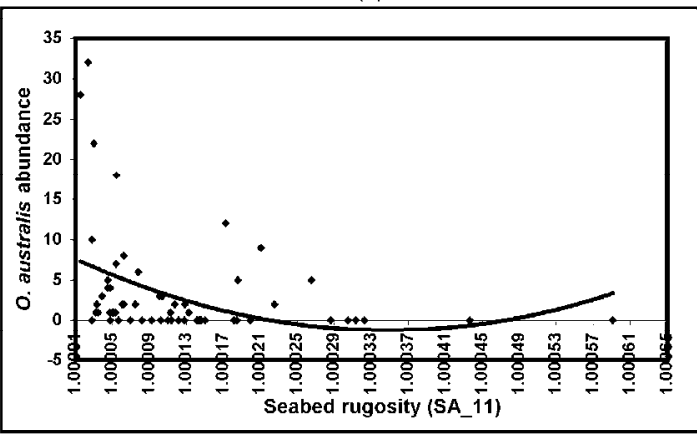

(d)

Figure S2: Sample values fitted with curves (see section 3.3.1) for individual species; (a): seabed disturbance and the abundance of $D$. dentate; (b): \%Caco3 and the abundance of $R$. robustus; (c): seabed disturbance and the abundance of $R$. robustus; (d): seabed rugosity and the abundance of $O$. australis; (e): habitat type and the abundance of $N$. inornata. 